\title{
The devil is in the details: Managing chest drains and interpreting negative randomized trial data
}

\author{
Eric Lim, MB ChB, MD, MSc (Biostatistics), FRCS (C-Th)
}

\author{
From the Academic Division of Thoracic Surgery, The Royal Brompton Hospital, London, United Kingdom. \\ Disclosures: The author's institution has received research grants and the author has received advisory board and \\ speaker fees from Medela (Zug, Switzerland). \\ Received for publication Sept 11, 2015; accepted for publication Sept 11, 2015. \\ Address for reprints: Eric Lim, MB ChB, MD, MSc (Biostatistics), FRCS (C-Th), Academic Division of Thoracic \\ Surgery, The Royal Brompton Hospital, Sydney St, London SW3 6NP, United Kingdom (E-mail: e.lim@rbht. \\ nhs.uk). \\ J Thorac Cardiovasc Surg 2015;150:1252-3 \\ $0022-5223 / \$ 36.00$ \\ Copyright (c) 2015 by The American Association for Thoracic Surgery \\ http://dx.doi.org/10.1016/j.jtcvs.2015.09.037
}

No single aspect of postoperative care in general thoracic surgery is subject to more variation than the management of chest drains. The issue is critically important because the duration of a patient's chest tube strongly influences length of hospital stay, patient mobility, and the patient's comfort during the duration of the admission. Yet almost all thoracic surgeons and institutions manage chest drains differently.

The decision for chest drain removal can be influenced by countless factors, but ultimately the decision is made on 2 main safety criteria: air and fluid. Some aspects of the decision-making process appear well rationalizedsurgeons do not want to remove drains when significant air leak is present (to prevent pneumothorax) or when there is too much fluid drainage (to prevent pleural effusion). When you drill down, the variation in decision making stems from differences in what levels of air leak and fluid output are considered safe. This is often not considered against the benefits of early removal with respect to patient comfort, mobility, and the length of hospital stay.

A long way from the days of the underwater seal, wall suction, patient confinement to a length of a suction tubing, and getting down on hands and knees to bubble count, today's drains offer portable suction, mobility, continuous digital quantification of air leak and fluid drainage, and the ability to consider chest drain removal in the presence of an air leak.

It is pertinent and timely that the randomized trial results reported by Gilbert and colleagues ${ }^{1}$ compare length of stay between patients randomized to digital drainage and underwater seal management policies. The authors used a fluid limit of $<250 \mathrm{~mL}$ (in both trial arms) and an air leak limit of $<40 \mathrm{~mL} / \mathrm{min}$ on suction or $<20 \mathrm{~mL} / \mathrm{min}$ on gravity mode cutoff in the digital drain arm compared with the absence of bubbles in the underwater seal arm. They found no statistically significant differences in length of hospital stay.

The first step in appraising a negative trial result is review of the sample size calculation.* This is often mistakenly

\footnotetext{
* The sample size calculation is an important determinant to guard against the probability of a type 2 error in a trial concluding no difference (of statistical significance) when a true difference exists.
}

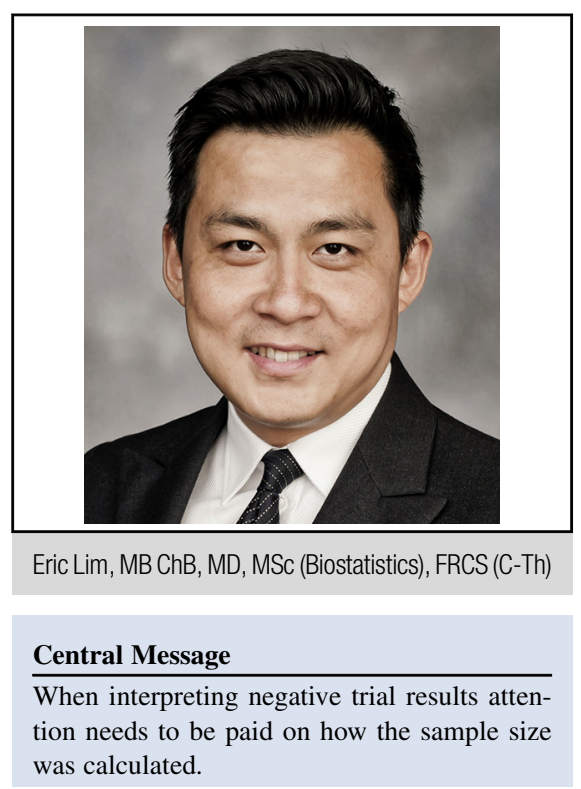

See Article page 1243.

referred to as a "power calculation" because the power is predetermined (usually at $80 \%$ or $90 \%$ ) by the trial statistician and the sample size is the actual number calculated. Apart from power, variables in the equation include level of statistical significance (usually set at .05 , but can be 1-tailed or 2-tailed), the variance of the data, and the minimal important difference. Smaller sample sizes will result from studies stipulating lower power, higher levels of statistical significance, 1-tailed $P$ values, smaller variances, and larger minimal important difference.

Whereas power and significance are usually set at conventional levels and variance is simply reported by statisticians, readers need to understand the meaning of a 1-tailed $P$ value and evaluate if they agree on the importance of a 1-day difference as used by Gilbert and colleagues, ${ }^{1}$ because these are the 2 measures that are decided by the clinician. A 1-tailed $P$ value usually halves the sample size required and tests the hypothesis that an intervention is "not worse." It is often used in studies comparing drugs of the same class to demonstrate safety (ie, the outcome of using a new drug is not worse than the outcome of using an existing drug). Strictly speaking, the reporting of the outcomes should be on the same vein in that the intervention is not worse as opposed to no difference (which implies a 2-way outcome and requires formal 2-tailed $P$ value when calculating a sample size). 
In my opinion, a specified minimum difference of 1 day is entirely reasonable, but hospital stay as a primary outcome presents a number of difficulties for sample size calculations. The distribution is almost always right-skewed and (straightforward) sample size calculations are usually based on normally distributed data. Astute readers will note that the parameter for sample size in the study by Gilbert and colleagues ${ }^{1}$ was presented as a median (nonparametric) length of stay of 5 days with a standard deviation (parametric) of 1.78 days. The Journal has a number of expert reviewers who identified the importance of sample size calculations and understood the difficulties associated with nonparametric outcomes. Hence the authors were advised to check their results using time-to-event analysis (Kaplan-Meier plots and log-rank testing is nonparametric and does not rely on any assumptions regarding the distribution of data). The authors report this information in their Figures 3 and 4.

One cannot underestimate the amount of effort, time, and money required to conduct a randomized trial. Ultimately the authors are to be congratulated for increasing the evidence base in thoracic surgery and editors and reviewers alike were reasonably satisfied that the conclusion was in line with the methods and results.

Where do we go from here? Our institutional experience suggests that air leak is the dominant factor influencing drain removal during the first 24 hours and fluid criteria are used thereafter (note the 1-day difference in the identification of an air leak resolution in the study by Gilbert and colleagues ${ }^{1}$ ). Unless we continue to push forward with trials using more permissive fluid criteria, the amount of fluid drainage is likely to be a dominant influence on chest drain duration (and length of stay) and we would not be able to accurately evaluate any influence of digital drains using air leak criteria.

\section{Reference}

1. Gilbert S, McGuire AL, Maghera S, Sundaresan SR, Seely AJ, Maziak DE, et al Randomized trial of digital versus analog pleural drainage in patients with or without a pulmonary air leak after lung resection. J Thorac Cardiovasc Surg. 2015; 150:1243-51 